\title{
Estudio de patentes sobre tecnologías para tratamiento de agua y el agua residual ${ }^{1}$
}

\section{A study of patenting on water and wastewater treatment technologies}

\author{
Odonel GONZÁLEZ-CABRERA² \\ Taimi RUIZ-PÉREZ ${ }^{3}$ \\ Mirelys CLARO-PÉREZ2 \\ Nancy María PÉREZ-PINO² \\ Grisel PÉREZ-GÁLVEZ² \\ Lisbey COLLAZO-ALFONSO²
}

\section{Resumen}

Se presenta un estudio de patentes sobre la temática tratamiento de agua y agua residual. Se localizan y descargan más de 2.500 documentos de patentes relacionados con la temática. Estos se procesan en software al efecto y herramientas de procesamiento. Se extraen indicadores de patentes para esta tecnología con alto valor para actividades comercialesy de investigación, tales como tecnologías emergentes sobre tratamiento de agua, países, entidades e inventores líderes a nivel mundial según el resultado de la tecnología patentada. Se presenta la evolución de las tecnologías de tratamiento del agua y el agua residual en los últimos veinte años a nivel mundial según el registro de las patentes. La entrega de todo el material se realiza en una herramienta de visualización que logra hacer más atractivo el servicio, tener un producto más amigable y cómodo para el uso, constituyendo una información de alto nivel de actualización y eficacia para la toma de decisiones de una empresa de proyectos e investigaciones hidráulicas.

Palabras ilave: Documento de patente. Indicadores de patentes. Tratamiento de agua.

\begin{abstract}
The aim of the study is to discuss the patenting of water and wastewater treatment technologies. More than 2.500 patent documents related to the topic were found and downloaded. The documents were analyzed by a software and processing tools. Patent indicators for the technology with high-value business and research were determined such as water treatment, countries, institutions and worldwide leader inventors according to the patented technology. The worldwide development of water and wastewater treatment technologies over the past twenty years is presented according to the record of patents. The delivery of all materials is done in a visualization tool that manages to make service more attractive, it is a friendlier and more comfortable product to use that offers a high-level update and effectiveness of information for decision making in a hydraulic project and research business.

Keywords: Patent document. Patent indicators. Water treatment.

\footnotetext{
1 Trabajo presentado en el VII Seminario Internacional sobre Estudios Cuantitativos y Cualitativos de la Ciencia y la Tecnología "Prof. Gilberto Sotolongo Aguilar en XIII Congreso Internacional de Información - INFO' 2014. Habana, Cuba.

2 Centro de Información y Gestión Tecnológica de Villa Clara. Marta Abreu \#55 entre Villuendas y Sayas, 50100, Santa Clara, Cuba. Correspondencia a nombre de/Correspondence to: O. GONZÁLEZ-CABRERA.E-mail:<ogonzalez@ciget.vcl.cu>.

3 Hospital Docente Gineco-obstétrico"Mariana Grajales". Santa Clara, Cuba.

Recibido el día 21/5/2014 y aceptado para su publicación el 12/9/2014.
} 


\section{Introducción}

El agua es un recurso fundamental para la vida tanto de los seres humanos como del resto de las especies de la tierra. Con un planeta cada vez más poblado y el cambio climático haciendo estragos en muchas partes del mundo, la gestión, conservación y acceso al agua en buenas condiciones es cada vez más difícil sobre todo en los países en desarrollo (Alemany, 2013).

El 59\% del consumo total de agua en los países desarrollados se destina a uso industrial, el 30\% a consumo agrícola y un $11 \%$ a gasto doméstico, según se constata en el primer informe de Naciones Unidas sobre el desarrollo de los recursos hídricos del mundo (Fernándes-Alba et al., 2006). Conscientes de la problemática en torno a este recurso, Naciones Unidas trabaja para conseguir que todos los ciudadanos del mundo puedan tener acceso al agua potable y condiciones de saneamiento dignas. Para ello el período comprendido entre 2005 y 2015 se declaró el Decenio Internacional para la Acción "El agua, fuente de vida". Dentro de las líneas de actuación de este proyecto, la Asamblea General de la Organización de Naciones Unidas (ONU) aprobó que el 2013 fuera el " Año Internacional de la Cooperación en la Esfera del Agua", en su resolución 65/154 con el objetivo de sensibilizar tanto sobre el potencial de una mayor cooperación como sobre los desafíos que afronta la gestión del agua debido a una mayor demanda y lo que ello implica para el acceso, la asignación y los servicios. El Año pondrá en valor la historia de las iniciativas exitosas de cooperación en materia de agua e identificará los temas emergentes y más urgentes.

El director general de la Organización Mundial de la Salud (OMS) Dr. Lee Jong-Wook plantea que el agua y el saneamiento son unos de los principales motores de la salud pública. Suelo referirme a ellos como "Salud 101", lo que significa que en cuanto se pueda garantizar el acceso al agua salubre y a instalaciones sanitarias adecuadas para todos, independientemente de la diferencia de sus condiciones de vida, se habrá ganado una importante batalla contra todo tipo de enfermedades (Wikipedia 2013).
Para poder contar con estos elevados fines debemos contar con los conocimientos de las tecnologías existentes para el tratamiento del agua y el agua residual, saber quiénes las han generado (inventores), quienes son sus dueños (titulares de las patentes), en que países están localizadas estas personas, empresas o centros de investigación (tema bloqueo y otros), nivel de obsolescencia de la tecnología y otros.

El tratamiento de agua y aguas residuales consiste en una serie de procesos físicos, químicos y biológicos que tienen como fin eliminar los contaminantes físicos, químicos y biológicos presentes en el agua para el uso humano, así como los efluentes de este. La tesis fundamental para el control de la polución por aguas residuales ha sido tratar las aguas residuales en plantas de tratamiento que hagan parte del proceso de remoción de los contaminantes y dejar que la naturaleza lo complete en el cuerpo receptor. Para ello, el nivel de tratamiento requerido es función de la capacidad de auto purificación natural del cuerpo receptor.

Por otro lado podemos exponer que existe un tipo de información tecnológica especial contenido en documentos llamados patentes de invención a los cuales por disímiles causas no se les presta la atención debida a la hora de resolver problemáticas como podría ser el tratamiento y potabilización de aguas. Las patentes constituyen una forma de proteger los inventos que desarrollan las empresas, instituciones o personas y que cumplan con los requerimientos de novedad mundial, actividad inventiva y aplicabilidad tecnológica; esta protección la otorga una oficina estatal y consiste en otorgarle el derecho de monopolio para su venta, producción, ofrecimiento de licencia, etc. Pero a la vez obliga al titular a rebelar todos los detalles técnicos de su invención y es precisamente este hecho el que aprovechamos para extraer la información precisa y actualizada (estos documentos se publican por las oficinas de patentes en un plazo posterior solo de 18 meses posterior a su presentación) de cualquier rama de la tecnología. También se realiza el procesamiento de la totalidad de documentos recuperada estadísticamente para extraer numerosos indicadores de patentes útiles para la toma de decisiones en las más disímiles disciplinas: vigilancia y transferencia tecnológica, búsqueda de potenciales, socios comerciales, encontrar la solución a 
problemas que tenemos y que alguien en el mundo ya solucionó, encontrar nichos tecnológicos para investigación, etc. La finalidad declarada del sistema de patentes es fomentar la invención y el progreso técnico facilitando un plazo temporal de exclusividad sobre la invención a cambio de su divulgación. Al ofrecer protección y exclusividad, la patente constituye un instrumento político cuya finalidad es alentar a los inventores a que inviertan en investigación y en el trabajo de innovación posterior que conseguirá que esas invenciones tengan una aplicación práctica (Organización para lá Cooperación y el Desarrollo Económicos, 2009).

Aprovechando el poder y facilidades que aporta el desarrollo de las Tecnologías de la Información y las Comunicaciones (TIC), se han desarrollado softwares para convertir la información de patentes en información gráfica clara o mapas, para permitir que el analista tenga una visión de los datos, saquen conclusiones y minimicen los riesgos en la hora tomar decisiones (Keim, 2002). También se ha designado como patinformática, a la ciencia de análisis de información sobre patentes para descubrir relaciones y tendencias que serían difíciles de ver cuando se trabaja con documentos de patentes de uno en uno (Trippe, 2003).

Basándonos en todo lo anterior expuesto realizamos el estudio sobre las tecnologías para el tratamiento y potabilización de agua para la Empresa de investigaciones y proyectos hidráulicos de Villa Clara. Presentando como objetivo principal: Fundamentar la toma de decisiones de una empresa de investigación y proyectos hidráulicos en temáticas de vigilancia tecnológica y transferencia de tecnología sobre tecnologías de tratamiento de agua y agua residual.

\section{Métodos}

Se implementan estrategias de búsqueda en la base de datos de patentes europea Esp@cenet (European Patent Office, 2013) bajo la temática de tratamiento de agua y agua residual. La estrategia combina los diferentes campos de búsqueda (palabras claves, fechas, clasificaciones, nombres) para recuperar un número de documentos manejables y sin que se excluyan los principales resultados a nivel mundial en la temática solicitada. Se trazó la siguiente estrategia de búsqueda:

Palabras claves en título: "Waste water"

Palabras claves en título o resumen: Treatment

Fecha de publicación: 2006-2012

Código de la Clasificación Internacional de Patentes (CIP): CO2F

C: Química

02F: Tratamiento del agua, agua residual, de alcantarilla o fangos

Cantidad de documentos de patentes: 2826

Base de datos: Esp@cenet

La totalidad de los datos bibliográficos se procesa en software de procesamiento de patentes desarrollado por el Centro de información y Gestión tecnológica de Villa Clara. Se extraen indicadores de patentes. Luego se completan documentos tales como: datos sobre el servicio, estrategias implementadas, breve introducción a la temática en cuestión, comportamiento de los indicadores de patentes, los resúmenes de todas las patentes procesadas y perfiles tecnológicos de las principales entidades generadoras de patentes en la temática. Utilizamos la base de datos (An open..., 2013) que actualmente cuenta con más de 10 millones de documentos de patentes incluidos los registros de las bases de Estados Unidos de América, Australia, Europa y World Intellectual Property Organization (WIPO). También nos hemos auxiliado temporalmente de Boliven Pro (Patents, 2013) procesador de patentes on line de Cambridge IP de la Universidad de Cambridge del Reino Unido y los directorios de patentes patentmaps (Wordwide..., 2013) y (Intellectual Property Exchange, 2013). Estas herramientas nos ayudan a de alguna manera validar el resultado que se obtiene con el software. Finalmente con este volumen de información se conforma el boletín en la herramienta de visualización desarrollado por el Centro de información y Gestión tecnológica de Villa Clara, en el cual permite manejar de forma fácil y amigable tan gran volumen de información.

Para la identificación de las tecnologías emergentes nos apoyamos en la CIP. Según la guía de la CIP, una invención se incluye en un grupo de la clasificación según su función o naturaleza intrínseca o 
bien por su campo de aplicación. Por tanto, éste es un sistema de clasificación combinado por funciones y aplicaciones en el que prevalece la aplicación. Una patente puede contener diversos objetos técnicos y ser asignada en consecuencia a varias clases CIP; estos códigos de la clasificación se publican en los documentos de patente. De manera global el comportamiento de las tecnologías para el tratamiento de aguas residuales es el siguiente:

En la CIP la clasificación C02F relaciona los documentos de patentes relacionados con tratamiento del agua, agua residual, de alcantarilla o fangos. Seguidamente a estas siglas el numero 1 indica tratamientos en general, el número 3 distingue a los tratamientos biológicos y el numero 9 hace alusión a tratamientos en varias etapas.

\section{Resultados}

Apoyados en las propiedades estadísticas de los datos de patentes que están determinadas por sus características jurídicas y su aplicación práctica en el ámbito económico, puesto que influyen en qué invenciones se protegen, quién las hace, qué información se hace pública (y, por tanto, accesible a los profesionales de la estadística) y qué importancia tienen las patentes para la industria, se extraen numerosos indicadores que nos ayudan en las más disímiles tareas del quehacer de una empresa de investigaciones y proyectos de obras hidráulicas.

Los indicadores basados en patentes constituyen una herramienta única, y en ocasiones la única disponible para seguir el ascenso de las tecnologías emergentes. El primer indicador nos entrega el comportamiento en el tiempo del registro de patentes, visualizando una pendiente creciente en esta tecnología, generándose gran cantidad de patentes cada uno de años siendo objeto de las pesquisas. Desde el año 2007 hasta el 2011 se presentan registros por encima de los 400, especial destaque para el 2010 (467). Este comportamiento muestra el interés elevado por esta temática dado la problemática de la contaminación de las aguas y la necesidad de agua para los más diversos usos.

Seguidamente se identifican a nivel mundial los países líderes en el registro de patentes en esta tecnología. Este indicador se toma de la fecha de prioridad; porque esta es la primera fecha de presentación de una solicitud de patente en cualquier lugar del mundo para proteger una invención y que por lo general se deposita en la oficina de patentes del país del solicitante. Es la más cercana a la fecha de la invención. Además de la cuestión práctica de este dato, incluye la sigla del país de prioridad.

Las patentes se utilizan para comprobar el rendimiento tecnológico de los países. En comparación con otros indicadores de producción, como pueden ser las publicaciones, las patentes constituyen un indicador más adecuado de las actividades más cercanas al desarrollo tecnológico. Ayudan a rastrear el liderazgo o posicionamiento en un campo o área determinados de la tecnología (por ejemplo, índices de ventajas tecnológicas reveladas) y los cambios que se producen con el tiempo. Como indicadores del rendimiento tecnológico, es decir, del nivel de especialización o fortaleza tecnológica de un país, las patentes ayudan a los gestores de políticas a identificar los puntos fuertes y débiles de sus sistemas de innovación nacionales.

China constituye abrumadoramente el país mayor productor y de mayor registro de las patentes (1.593 para 56,00 \% del total), seguido de Japón (463 y $16,00 \%$ ), Corea del Sur (338 y 12,00\%) y Alemania (106 y 3,75\%) entre otros (Figura 1).

Importante es identificar las firmas más destacadas, a este fin tributa un dato contenido en documento de patente llamado titular de la patente, esto no es más que la persona o personas, físicas o jurídicas, a quienes el inventor transfiere jurídicamente en su totalidad o de forma limitada los derechos dimanantes de una patente. Este puede ser un centro de Investigación y Desarrollo; una universidad, una empresa o una persona natural (generalmente el mismo inventor).

Es notable en los últimos años el impacto de las universidades en este particular, siendo verificable recopilando datos de las patentes que han obtenido, las citas que generan, etc. En un número cada vez mayor de países, agencias o ministerios encargados de aportar financiación, estos utilizan el número de patentes para evaluar el rendimiento de las instituciones académicas o de investigadores determinados. 


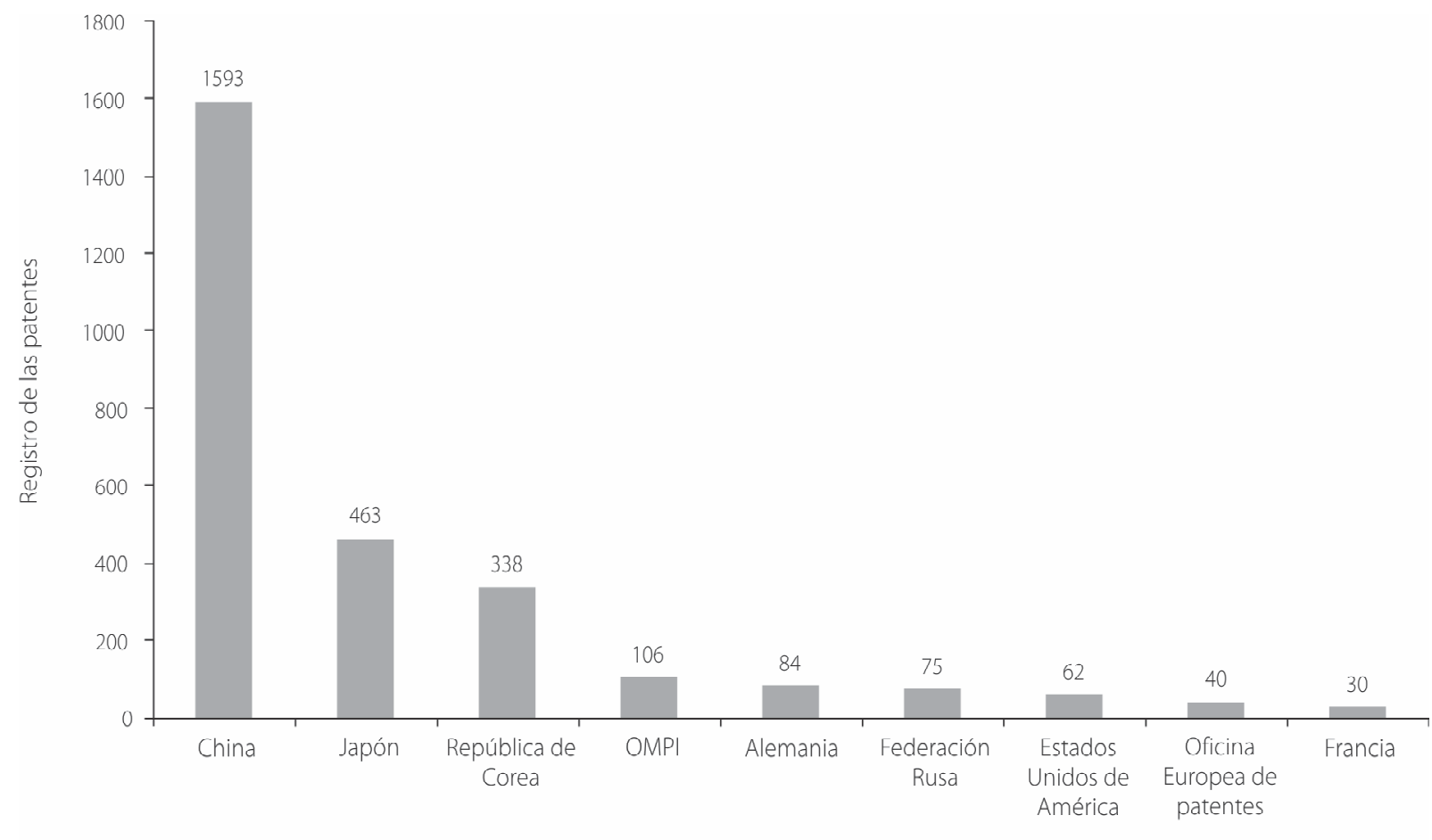

Paises líderes

Figura 1. Países líderes.

Note: OMPI: Organización Mundial de la Propriedad Intelectual.

Fuente: Elaboración propia, 2013.

Las patentes incluyen información sobre las actividades y el rendimiento inventivo de las multinacionales. A través de las direcciones de los solicitantes y los inventores, es posible dibujar los patrones y medir la intensidad de la coinvención internacional. El análisis de los titulares líderes en la temática brindó como resultado que existe gran variedad de firmas implicadas en la producción de patentes relacionadas al tema (Figura 2). Principalmente son como se exponía más arriba coincidente con las tendencias mundiales, universidades y reconocidas transnacionales.

Los inventores son personas físicas, por lo general empleados del solicitante de la patente. En Estados Unidos, los inventores son los solicitantes. No obstante, también es posible que una misma persona sea el inventor y el solicitante (por ejemplo, el caso de los inventores independientes).

Los investigadores líderes a nivel mundial resultaron el señor Anil Jha de United States filter
Corporation con 50 solicitudes y Nobuyuki Ashikaga de Toshiba con 49 solicitudes. Un indicador muy importante del registro de patentes a nivel mundial es poder identificar dentro de un proceso tecnológico cuales son las variantes con mayor número de registros y su evolución en el tiempo.

En cuanto a las tecnologías de tratamiento del agua con más solicitudes utilizando los códigos de la CIP se detectan los siguientes (Tabla 1).

Estas son variantes tecnológicas que en el periodo 2006-2013, con más solicitudes presentan, por lo que afirmamos que son las tecnologías en pleno desarrollo o emergentes.

Se extraen otros indicadores importantes como son la evolución de cada una de estas variantes tecnológicas a nivel de subclase por año. Se observa una casi totalidad de registros en la subclase $\mathrm{C} 02 \mathrm{~F}$ (Tratamiento del agua y el agua residual) con presencia en todos los años estudiados, seguido de B01D (Métodos físico-químicos de separación) con presencia de más de 
diez registros por año y llegando a 23 en el 2007. Se identifican dentro de los tratamientos biológicos los más destacados, siendo estos los tratamientos que emplean fangos activados (751) y los que usan microorganismos (624). Dentro de los de varias etapas se destacan los que al menos incluyen en una etapa un tratamiento biológico y los que incluyen uno químico (631). Se logra obtener un indicador muy importante pues combina temáticas, años de solicitud y cantidad de documentos, siendo un complemento de los indicadores anteriores. Se incluyen además los perfiles de firmas de las firmas líderes y de personalidad de los inventores líderes. Se incluyen los

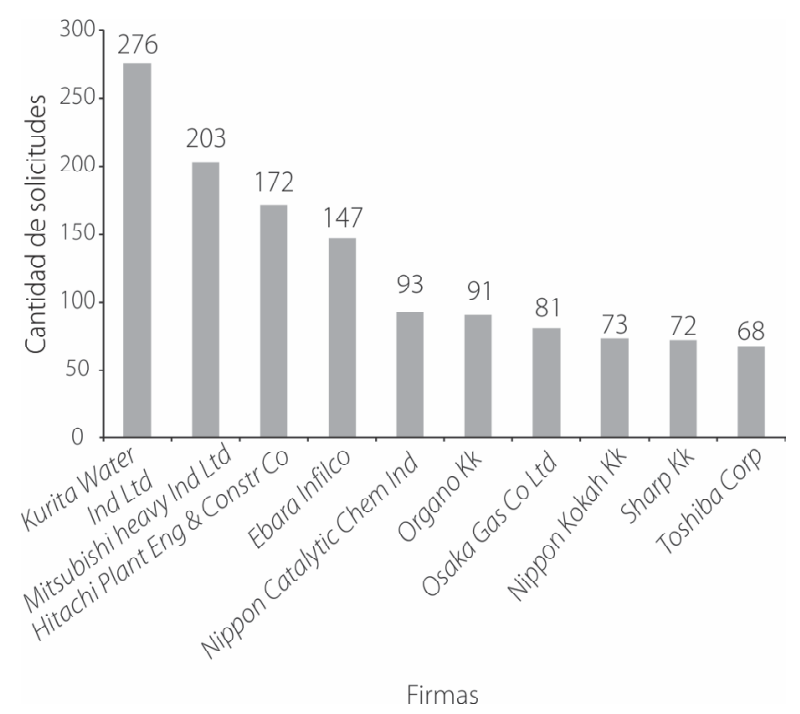

Figura 2. Firmas líderes.

Fuente: Elaboración propia, 2013. resúmenes de la totalidad de las patentes y los textos completos de las más destacadas, tomando como parámetro para el grado de importancia las citaciones en posteriores documentos. Toda la información se presenta en una herramienta informatizada de presentación donde se incluyen los siguientes documentos: Breve descripción del servicio, la estrategia implementada, un informe basado en información no patente del tema de la búsqueda, el comportamiento de cada uno de los indicadores de patentes acompañado de la explicación del mismo, el resumen de las patentes, las patentes a texto completo de los más importantes, los perfiles de firmas y personalidades (Figura 3).

\section{Discusión}

En la literatura se refieren numerosos artículos sobre el tratamiento del agua. Durante años se ha utilizado el cloro para la desinfección del agua con buen resultado. No obstante, el cloro reacciona con compuestos orgánicos presentes en las aguas y produce compuestos organoclorados y trialometanos, los cuales tienen efectos mutagénicos y carcinogénicos. Además, se reportan microorganismos patógenos resistentes al cloro (Fernández García, 2013). Sin embargo, el empleo del ozono es una alternativa segura y eficaz de tratamiento de aguas, no se reportan los inconvenientes de la cloración. En este sentido se ha incrementado en las últimas décadas dado su alto poder oxidante y su elevado efecto germicida de amplio espectro contra bacterias, hongos, virus y quistes de parásitos, junto a la

Tabla 1. Tecnologías más registradas.

\begin{tabular}{llc}
\hline $\begin{array}{l}\text { Código de la Clasificación } \\
\text { Internacional de Patentes }\end{array}$ & \multicolumn{1}{c}{ Descripción } & $\begin{array}{c}\text { Cantidad de documentos } \\
\text { encontrados }\end{array}$ \\
\hline C02F1/44 & Tratamiento del agua por diálisis ósmosis u ósmosis inversa & 287 \\
C02F1/52 & $\begin{array}{l}\text { Tratamiento del agua por floculación o precipitación de las impurezas en } \\
\text { suspensión }\end{array}$ & 248 \\
C02F1/28 & Tratamiento del agua por absorción o adsorción & 248 \\
C02F1/32 & Tratamiento del agua por irradiación por luz ultravioleta & 158 \\
C02F1/50 & Tratamiento del agua por adición o empleo de un germicida o por tratamiento & 148 \\
C02F1/78 & oligodinámico & 145 \\
\hline
\end{tabular}

Fuente: Elaboración propia, 2013. 


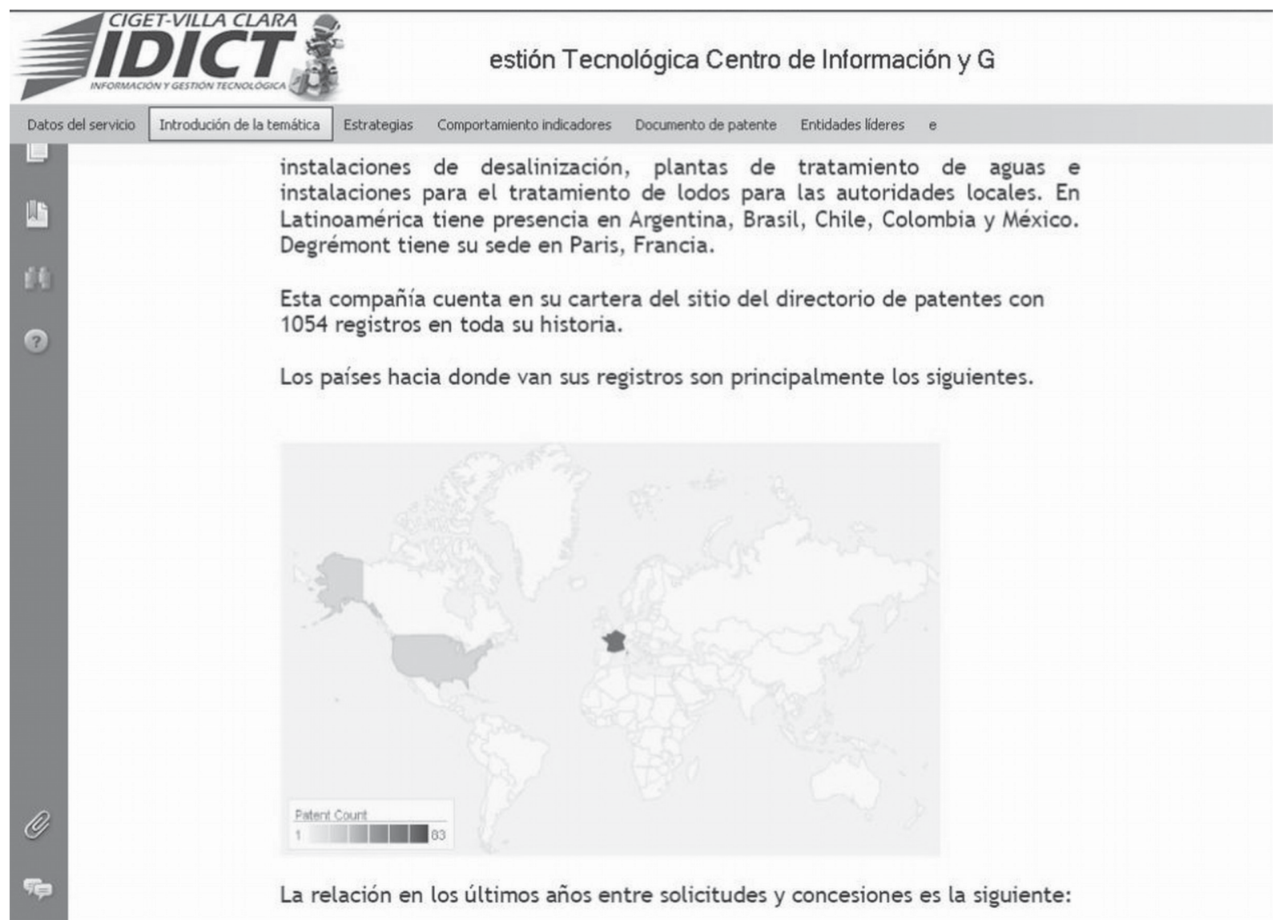

Figura 3. Herramienta para visualizar la información.

Fuente: Wordwide... (2013).

producción de ozonizadores más compactos y eficientes. Se menciona la aplicación del método de oxidación por medio de ozono en procesos de potabilización de aguas, tratamientos de aguas residuales de diferente procedencia y para el tratamiento de agua de piscinas, también en aplicaciones a usos o procesos en los que se requiera un agua de elevada calidad como en la Industria farmacéutica y alimenticia. Este estudio corrobora la validez de esta tecnología y su factible uso detectado como una de las tecnologías emergentes en el presente estudio.

En las aguas que no se utilizarán en el consumo humano son otras las tecnologías más factibles de emplear, como pueden ser diferentes tratamientos biológicos. Se refiere la utilización de pasto vetiver, a pequeña escala el objetivo es estudiar el tratamiento complementario de aguas residuales en una planta de residuales de fábrica de pepsi-cola (Scavo et al., 2013). Este estudio no analiza otras tecnologías basadas en tratamientos biológicos también muy factibles a emplear. Otro estudio realizado en la Universidad de Stuttgart, Alemania; contando con unas noventa páginas, para el tratamiento de las aguas y su reuso en la capital del Perú específicamente del área metropolitana (McElhinney, 2012) se abordan las alternativas en cuanto a tecnologías para aplicar y no se menciona ni en las fuentes de información consultadas y en las tecnologías comparadas nada acerca de patentes que describen las tecnologías y que son la fuente primaria muchas veces.

Otro estudio (Radjenovic et al., 2009) muestra los resultados del uso de tecnologías detectadas como la osmosis inversa Reverse Osmosis (RO) utilizada para la eliminación de residuales farmacéuticos. Este estudio tampoco menciona la información contenida en los 
documentos de patentes como basamento teórico en la toma de decisiones a la hora de emplear un método u otro.

En otro documento (Reverse..., 2009) se plantea que cuando sólo se necesita un pequeño volumen de agua tratada, aproximadamente 0-10 galones por día, la osmosis inversa (RO) es normalmente el proceso más flexible y rentable de tratamiento disponibles para uso doméstico privado.

Por otro lado se plantea en cuanto al uso de tecnologías de oxidación con ozono (Ozone..., 2011) viable como desinfectante para matar microorganismos en el aire y el agua. Muchos spas y jacuzzis usan ozono para mantener el agua libre de algas y bacterias dañinas. El ozono también se utiliza con fines industriales y de fabricación, así como el blanqueo y agente desodorante. Las plantas de tratamiento de agua que fueron construidas en 1950 y 1960, respectivamente, se están actualizando para satisfacer las crecientes demandas de agua, a lo largo de las regulaciones de agua más estrictas federales y estatales, con el objetivo final de mejorar la calidad del agua. Históricamente, el cloro ha sido utilizado con eficacia en estas plantas de tratamiento de agua a desinfectar el agua potable. Sin embargo, con los avances en el tratamiento del agua, ha sido determinado que la desinfección con ozono es rentable y resultará en agua de mejor calidad.

Existe en la literatura numerosos estudios sobre tecnologías para el tratamiento de agua y agua residual, pero ninguno hace referencia a indicadores de patentes y documentos de patentes que describen las invenciones que solucionan las diferentes problemáticas tecnológicas que esta esfera presenta. Algunos estudios corroboran el grado de maduración de las tecnologías detectadas en nuestro estudio como tecnologías en auge, lo que indica la precisión del estudio de las patentes a la hora de localizar tecnologías para aplicaciones en transferencia de tecnología.

Indicadores de patentes como las firmas líderes nos sirven para analizar posibles socios comerciales, adquisición de licencias de patentes y otros tipos de negociaciones. Los demás indicadores ayudan a tener una visión global de la situación de desarrollo tecnológico en la materia que queramos investigar.
Es de vital importancia en las temáticas de información la manera que se presenta esta. A este efecto las tecnologías de la información y la comunicación juegan un papel primordial ofreciéndonos herramientas que a la distancia de un clic nos permite acceder a la información extractada y de alta elaboración como fungen los servicios de información que ofrecemos. El poder contar con herramientas de procesamiento, presentación y representación de la información logra hacer más atractivo el servicio, tener un producto más amigable y cómodo para el uso.

\section{Conclusión}

Se logra recuperar un número suficiente de soluciones tecnológicas contenidas en los documentos de patentes fundamentado por la estrategia de búsqueda trazada.

Se procesa la totalidad de los datos bibliográficos de las patentes utilizando para ello herramientas diseñadas en el centro de Información y Gestión tecnológica de Villa Clara y otras disponibles en la Web, extrayendo varios indicadores que permiten tomar decisiones en cuanto a tecnologías a utilizar en dependencia del uso posterior del agua y por supuesto el espacio disponible y los recursos financieros. También se identifican líderes mundiales en la generación de soluciones tecnológicas a la problemática en cuestión como es la firma japonesa Kurita Water Industries Limited y otras firmas e institutos de investigación con los que se puede negociar cualquier tipo de asociación. Se obtienen otros indicadores de actividad de países, inventores y otros de valor aplicable a actividades de centros de investigación y proyecto como patentes más citadas por otros autores lo que valoriza el documento como primario de una tecnología.

Toda la información recuperada es de gran utilidad para investigación y proyecto de plantas para tratamiento de agua y agua residual, cumpliendo así con los requisitos para ser la más valiosa fuente de información en la actividad de vigilancia tecnológica. Documentos de patentes relativos a equipos para decidir a la hora de proyectar equipamiento para tratamiento de agua y potabilización. Documentos relativos a procedimientos a la hora de decidir qué tipo de tratamiento emplear según su aplicación a tratamientos de aguas residuales de diferentes industrias. 
Alemany, S. Año internacional de la cooperación en la esfera del agua. 2013. Disponible en: <http://www.ecogestos.com/ 2013-ano-internacional-de-la-cooperacion-en-la-esfera-delagua/>. Acceso en: 15 enero 2013.

European Patent Office. 2013. Available from: <http://world wide.espacenet.com/advancedSearch?locale $=e n \_E P>$. Cited: Sept. 26, 2013.

Fernándes-Alba, A.R. et al. Tratamientos avanzados de aguas residuales industriales. Madrid: Universidad de Alcalá, 2006. (Vigilancia Tecnológica, v.2).

Fernández García, L.A. Tratamiento de aguas y aguas residuales con el empleo de ozono. Centro de investigaciones del ozono. 2013. Disponible en: <http://www.ozono.cubaweb.cu/acerca/ tratamiento.htm>. Acceso en: 22 oct. 2013.

Intellectual Property Exchange. 2013. Available from: <www.ipexl.com/>. Cited: May 19, 2013.

Keim, D.A. Information visualization and visual data mining. IEEE Transactions on Visualization and Computer Graphics, v.8, n.1, p.18- 67, 2002.

McElhinney, K.J. Auditing water resources for application to water-sensitive urban design: A case study in the Lima (Perú) metropolitan area. 2012. Available from: <http://www.limawater.de/documents/kmcelhinney_tesis.pdf $>$. Cited: Dec. 17, 2013.

Open source for innovation cartography (An). Patent Lens. 2013. Available from: <http://www.lens.org/lens/>. Cited: Dec. 12, 2013.

Organización para lá Cooperación y el Desarrollo Económicos. Manual de estadísticas de patentes de la OCDE. 2009. Disponible en: <http://www.oepm.es/export/sites/oepm/comun/ documentos relacionados/Memorias de Actividades y_Estadisticas/EstadisticasOCDE/Estadisticas_OCDE.pdf>. Acceso en: 23 oct. 2013.

Ozone disinfection for drinking water Miramar and Alvarado water treatment plants. 2011. Available from: <http:// www.sandiego.gov/cip/pdf/ozonefaq.pdf>. Cited: Apr. 15, 2013.

Patents. CambridgIP. 2013. Available from: <http://www. boliven.com/patents/search?q=abstract\%3A\%28water+ treatment\%29\&query_type=>. Cited: May 20, 2013.

Radjenovic, J. et al. Rejection of pharmaceuticals in nanoltration and reverse osmosis membrane drinking water treatment. Water Research, v.42, n.14, p.3601-3610, 2008.

Reverse osmosis treatment for drinking water. New Hampshire: Departmente Environmental Services, 2009. Available from: <http://des.nh.gov/organization/commissioner/pip/ factsheets/dwgb/documents/dwgb-2-11.pdf>. Cited:May 7, 2013.

Scavo, M.; Rodriguez, O.; Luque, O. Estudio de un sistema de tratamiento de aguas residuales complementario, con pasto vetiver (vetiveria zizanioides I.), provenientes de una planta de producción de gaseosas, en villa de cura, estado Aragua. 2013. Disponible en: <http://www.vetiver.org/ICV4pdfs/BA17es. pdf>. Acceso en: 19 dic. 2013

Trippe, A.J. Patinformatics: Tasks to tools. World Patent Information, v.25, n.3, p.211-221, 2003.

Wikipedia. Agua potable. 2013. Disponible en: <http:// es.wikipedia.org/wiki/Agua_potable>. Acceso en: 18 feb. 2013.

Worldwide patent directory and citation statistics. Patentmaps. 2013. Available from: <http://www.patentmaps.com/ inventor/index.html>. Cited: May 26, 2013. 

